\title{
Downregulated myocardial connexin 43 and suppressed contractility in rabbits subjected to a cholesterol-enriched diet
}

\author{
Lung-Chun Lin ${ }^{1}$, Chau-Chung $\mathrm{Wu}^{1}$, Hung-I Yeh², Long-Sheng $\mathrm{Lu}^{3}$, Yen-Bin Liu ${ }^{1}$, \\ Shien-Fong $\operatorname{Lin}^{4,5}$ and Yuan-Teh Lee ${ }^{1}$
}

\begin{abstract}
${ }^{1}$ Division of Cardiology, Department of Internal Medicine, National Taiwan University Hospital, Taipei, Taiwan; ${ }^{2}$ Departments of Medical Research and Internal Medicine, Mackay Memorial Hospital, Mackay Medicine, Nursing and Management College, Taipei, Taiwan; ${ }^{3}$ Institute of Pharmacology, College of Medicine, National Taiwan University, Taipei, Taiwan; ${ }^{4}$ Division of Cardiology, Department of Medicine, Cedars-Sinai Medical Center, David Geffen School of Medicine, UCLA, Los Angeles, CA, USA and ${ }^{5}$ Department of Medicine (Cardiology) and Physiology and Physiological Science, David Geffen School of Medicine, UCLA, Los Angeles, CA, USA
\end{abstract}

\begin{abstract}
The effects of hypercholesterolemia on the myocardium per se include electrophysiological and mechanical alterations. Since gap junctions are essential in electromechanical coupling throughout the heart, we examined the correlation between the temporal expression of cardiac connexin 43 (Cx43), contractile function, and conduction velocity in cholesterol-fed rabbits. After a 12-week feeding period, serum cholesterol levels gradually increased $(P<0.001)$. In contrast, expression of cardiomyocyte $C \times 43$ protein progressively decreased $(60 \%$ reduction at 12 weeks, $P<0.001)$. Such a reduction was also demonstrated by immunoconfocal microscopy, which further showed redistribution of Cx43 gap junctions at the lateral cell membrane. The downregulation of Cx43 protein was associated with increased levels of Cx43 mRNA (3.5 -fold at 12 weeks, $P<0.001$ ) and phosphorylated c-Jun $\mathrm{N}$-terminal kinase (three-fold at 12 weeks, $P=0.001$ ). Functionally, although fractional shortening of the left ventricle remained unchanged throughout the feeding protocol, the cholesterol-fed rabbits had a reduced cardiac cycle-dependent variation of integrated backscatters, a decreased mitral ring systolic velocity, and an increased modified Tei index (all $P<0.001$ ), all of which indicated impaired intrinsic myocardial contractility and attenuated ventricular systolic performance. In Langendorff-perfused hearts of cholesterol-fed rabbits, decreased conduction velocity was observed $(P<0.005)$. Withdrawal of the cholesterol-enriched diet for 18 weeks restored the contractile parameters and Cx43 protein expression. These findings suggest that $\mathrm{Cx} 43$ is highly involved in the molecular mechanism of hypercholesterolemia-induced cardiac contractile dysfunction and dysrhythmias.
\end{abstract}

Laboratory Investigation (2005) 85, 1224-1237. doi:10.1038/labinvest.3700324; published online 15 August 2005

Keywords: connexin; contractility; echocardiography; gap junction; hypercholesterolemia

Hypercholesterolemia is a well-known cardiovascular risk factor, and its impact on coronary arteriosclerosis has been established by five decades of investigation. However, the effects of elevated serum levels of cholesterol on the myocardium per se have been less clear. We have observed that hypercholesterolemia can induce myocardial electrical remodeling, which was associated with prolonged action

Correspondence: Professor Y-T Lee, PhD, Division of Cardiology, Department of Internal Medicine, National Taiwan University Hospital, No. 7, Chung-Shan South Road, Taipei 10016, Taiwan. E-mail: ytlee@ha.mc.ntu.edu.tw

Received 30 December 2004; revised 19 June 2005; accepted 22 June 2005; published online 15 August 2005 potential duration, longer QTc intervals, increased repolarization dispersion, and increased vulnerability to ventricular fibrillation. ${ }^{1}$ The increased susceptibility to inducible ventricular arrhythmias has been also observed in mice with modulation of cardiac gap junction expression. ${ }^{2,3}$ The major function of cardiac gap junctions is to maintain efficient electric coupling for ensuring organized propagations of action potential that govern orderly contraction of the heart. ${ }^{4}$ Two principal, nonmutually exclusive, gap junction-related alterations have been reported in the diseased ventricle: disturbances in the distribution or organization of gap junctions (structural remodeling), and reduced levels of their major component, connexin 43 (Cx43). ${ }^{5,6}$ Alterations 
in the organization of cardiac gap junctions and expression of $\mathrm{Cx} 43$ can cause predisposition to arrhythmia, uncoordinated contraction, and overall diminished myocardial function in various pathologic conditions. ${ }^{5-9}$ Using chimeric mice created from Cx43-deficient stem cells and blastocysts, Gutstein et $a l^{10}$ have demonstrated that spatially heterogeneous Cx43 downregulation is indeed linked to disturbances in electromechanical function in terms of both irregular epicardial conduction patterns and contractile dysfunction. ${ }^{10}$

Dietary hypercholesterolemia has been also shown to induce contractile dysfunction independent of vascular disease, characterized by a decrease in the maximum rate of shortening and relaxation in a papillary muscle preparation, ${ }^{11}$ although this finding has not been confirmed in isolated intact hearts. ${ }^{12}$ Ultrasonic tissue characterization (UTC) with cardiac cycle-dependent variation of integrated backscatters (CVI) has been shown to be representation of intrinsic myocardial contractility and a potential indicator of early systolic dysfunction. ${ }^{13-15}$ Different structural components of the myocardium can influence its acoustic properties under different physiological and pathological conditions. Generated from the altered acoustic impedance mismatching between the myocytes' intracellular contents and the extracellular collagen networks during a cardiac cycle, ${ }^{16}$ the magnitude of CVI depends not only on the microstructural arrangement of myocytes embedded in a collagen matrix, ${ }^{17,18}$ but also on coordinated contraction of adjacent cardiomyocytes. Since previous studies have demonstrated both the electrophysiological and mechanical alterations of the myocardium in experimental hypercholesterolemia, this study was conducted. Using a series of time points, we studied the expression of Cx43 in such hearts, correlating contractile function detected by in vivo echocardiographic examination and conduction velocity measured using optical mapping of Langendorffperfused hearts. In addition, since activation of c-Jun N-terminal kinase (JNK), a stress-activated protein kinase, has been shown to cause rapid loss of Cx43 in cultured myocytes as well as induce cardiac dysfunction and premature death in transgenic animal models, ${ }^{19}$ the status of JNK was also examined.

\section{Materials and methods}

\section{Animals}

Control $(n=20)$ and cholesterol-fed (CF, $n=25)$ male New Zealand White Rabbits weighting 2.0$2.2 \mathrm{~kg}$ were used in this study. Control rabbits were maintained on standard calibrated chow (Purina 5321), and the CF rabbits were fed batch-matched standard chow supplemented with $0.5 \%$ cholesterol (Wako Co) and coconut oil (Yeali Co) for up to 12 weeks. $^{20}$ Every 3 weeks after initiation of the cholesterol-enriched diet, we executed echocardiography, examined the serum levels of total cholesterol and triglyceride, and killed five animals to harvest the hearts. After completing the 12-week feeding protocol, the residual five hypercholesterolemic rabbits continued feeding on standard chow for 18 weeks to study the effects of dietary cholesterol withdrawal (cholesterol-withdrawn, $\mathrm{CW}$ ). The investigation conforms to the Guide for the Care and Use of Laboratory Animals published by the US National Institutes of Health (NIH Publication No. 85-23, revised 1996).

\section{Echocardiography}

We performed echocardiography with a commercially available machine (SONOS 5500, Philips, Andover, MA, USA) equipped with a $12-\mathrm{MHz}$ phased-array probe. The rabbits were anesthetized with intramuscular injections of xylazine $(10 \mathrm{mg} / \mathrm{kg}$, Bayer) and ketamine (15 mg/kg, Parke-Davis). Through the parasternal short-axis view at the papillary muscle level, left ventricular wall thickness and fractional shortening were measured. The transmitral and aortic blood flow velocities were examined from apical views to record the peak velocity of early (E) and late (A) filling waves, deceleration time of $\mathrm{E}$ waves, $\mathrm{E} / \mathrm{A}$ ratio, and the aortic outflow peak velocity with time-velocity integral. By activating tissue Doppler imaging, peak velocities of the systolic (Sm), early (Em) and late (Am) diastolic waves were sampled at the lateral margin of the mitral annulus. A Doppler index of myocardial performance (Tei index), which is defined as the sum of isovolumic contraction time and isovolumic relaxation time divided by ejection time has been tested to correlate with invasive measurements of left ventricular systolic and diastolic function. ${ }^{21}$ This index is not influenced by heart rate or blood pressure. In this study, a modified Tei index was measured by tissue Doppler imaging (Figure 1, upper panel). This parameter has the advantage of being derived within the same cardiac cycle and is well correlated with the Tei index. ${ }^{22}$ All the Doppler echocardiograms were recorded with a sweep speed of $100 \mathrm{~mm} / \mathrm{s}$ and had an average of $\geq 5$ consecutive beats.

We performed UTC using the software package (Acoustic Densitometry) incorporated into the same machine as described in our previous studies. ${ }^{15,23}$ The grayscale images were displayed proportional to the integrated backscatters power with a dynamic range of $60 \mathrm{~dB}$. The parasternal short-axis image of the left ventricle was obtained at the level of papillary muscles and digitally stored on an optic disc with 60 frames of $33 \mathrm{~ms}$ time increments. A $21 \times 21$-pixel elliptical region of interest was placed in the mid-myocardium of the posterior wall and tracked manually to obtain the time-intensity curve of backscatters power off-line. The CVI was 

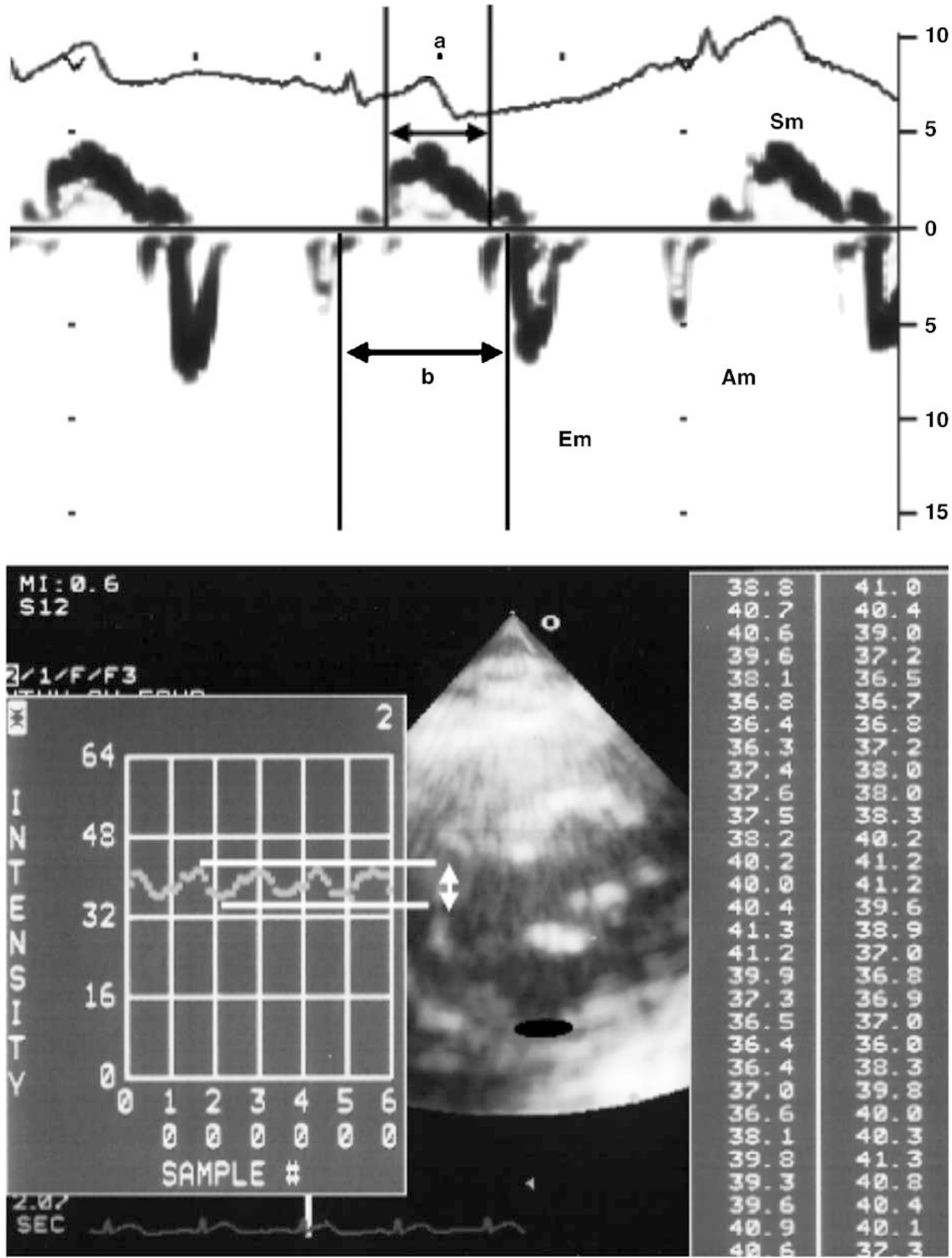

Figure 1 Upper panel: Doppler tissue imaging with sample volume on the lateral margin of mitral annulus to calculate the modified Tei index. $a$ is the duration of mitral annular systolic wave and $b$ is the time interval between the end and onset of mitral annular diastolic waves. The modified Tei index is calculated as $(b-a / a)$. Am = late diastolic wave; Em = early diastolic wave; Sm $=$ systolic wave. Lower panel: two-dimensional integrated backscatters image from parasternal short-axis view at the papillary muscles level. The power of integrated backscatters was measured at the posterior wall (black ovoid), and the magnitudes of cyclic variation were calculated as the differences between maximum and minimum values in a cardiac cycle (between arrowheads). 
calculated as the difference between the minimum and maximum backscatters power in each cardiac cycle, averaging $\geq 4$ consecutive beats (Figure 1, lower panel).

\section{Tissue Preparation and Pathology}

All procedures, as described previously, ${ }^{24}$ were under the regulation of the Republic of China Animal Protection Law (Scientific Application of Animals). To harvest the hearts, studied animals were anesthetized with intravenous pentobarbital $(100 \mathrm{mg} / \mathrm{kg})$ and retrogradely perfused with phosphate-buffered saline via the abdominal aorta. Blocks of myocardium from the posterior wall (corresponding to the region of interest during UTC) were excised and cryoembedded for subsequent histopathological examination. Hematoxylineosin and Masson trichrome stains were performed according to standard protocols. Immunolabeling of Cx43 was performed using a commercially available monoclonal antibody (Transduction Lab).

\section{Determination of Hydroxyproline}

We analyzed 15 samples (three frozen tissue blocks in each animal) from the control, CF (12 weeks) and CW groups. Hydrolysis of samples was carried out in $6 \mathrm{~N} \mathrm{HCl}$ at $110^{\circ} \mathrm{C}$ for $16 \mathrm{~h}$. After evaporating under a vacuum, hydroxyproline was measured using the colorimetric method described by Stegemann and Stalder. ${ }^{25}$ This method involves oxidation of hydroxyproline by chloramine-T (sodium $N$-chloro- $p$ toluenesulfonamide) and formation of a chromophore with $p$-dimethylaminobenzaldehyde. The absorbance was measured by a spectrophotometer at $550 \mathrm{~nm}$ within $45 \mathrm{~min}$.

\section{Immunoblotting}

The snap-frozen myocardium of each animal was homogenized, and protein was extracted with an alkaline extraction method described elsewhere. ${ }^{26}$ The protein was loaded with an equal amount $(20 \mu \mathrm{g})$ per lane for electrophoresis. After transfer to nitrocellulose, membranes were blocked and probed for $60 \mathrm{~min}$ with anti-Cx43 (1:250), anti-JNK (1:100; Santa Cruz Biotechnology), or anti-phosphoJNK (1:1000; Cell Signaling Technology). Membranes were washed and then incubated with an alkaline phosphatase-conjugated secondary antibody (1:5000; Pierce). The enzymatic activity was revealed with nitro blue tetrazolium/5-bromo-1chloro-3-indolyl phosphate (NTB/BCIP) solution (Pierce) and quantified by densitometry. For normalization of signals, reprobing was performed with anti- $\alpha$-actin (Chemicon) monoclonal antibody after stripping the membranes.

\section{Immunofluorescence Study and Confocal Microscopy}

For the morphometric analysis of gap junctional plaques, cryosections $10 \mu \mathrm{m}$ thick of three rabbits (three specimens for each animal) from each occasion of echocardiographic observation were immunostained and examined by confocal laser scanning microscopy (Leica TCS SP) as described previously elsewhere. ${ }^{8,24,27}$ For each specimen, 10 randomly selected areas were examined. Mouse anti-rabbit immunoglobulin conjugated to CY3 (Chemicon) was used to visualize immunolabeled Cx43. Each recorded image, parallel to the long axis of cells, consisted of $1024 \times 1024$ pixels $(0.24 \mu \mathrm{m}$ for each pixel) using a $\times 40$ objective lens and zoom 1.0 computer setting. Projection views of consecutive optical sections taken at $0.4-\mu \mathrm{m}$ intervals through the full thickness of the myocardium were recorded. Assisted by QWIN image analysis software (Leica), we measured (1) the proportion of total tissue area occupied by the Cx43 immunoreactive signal, as well as (2) the density (number/100 $\mu \mathrm{m}^{2}$ ) and (3) the size of discrete clusters of immunoreactive signal, which we defined operationally as individual gap junctions. ${ }^{7,24,27}$

\section{Isolated Rabbit Heart Preparation and Optical Mapping}

The hearts of CF (from another three animals after feeding for 12 weeks) and control $(n=6)$ rabbits were quickly removed under anesthesia, and Langendorff-perfused with $37^{\circ} \mathrm{C}$ Tyrode's solution. ${ }^{28}$ Coronary perfusion pressure was regulated between 80 and $95 \mathrm{mmHg}$. Optical mapping studies (Figure 6 ), as described previously, ${ }^{1,28}$ were performed to determine the conduction velocity (CV) at pacing cycle lengths (PCL) of 400, 300, and $200 \mathrm{~ms}$. For optical mapping, $5 \mu \mathrm{mol} / \mathrm{l}$ cytochalasin D (Sigma Inc.) was used as an electromechanical uncoupler and $0.5 \mu \mathrm{mol} / \mathrm{l}$ di-4-ANEPPS (Molecular Probes) was used as a voltage-sensitive dye. We mapped the left ventricular anterior wall $\left(20 \times 20 \mathrm{~mm}^{2}\right)$, acquiring $128 \times 128$ sites simultaneously at a sampling rate of 460 frames per second. We measured CV along three different directions (Figure 6b). The mean values of these CVs became the CV $(\mathrm{cm} / \mathrm{s})$ for that rabbit. ${ }^{28}$

\section{Reverse Transcription and Polymerase Chain Reaction Amplification}

Semiquantitative reverse transcription-polymerase chain reaction (RT-PCR) was used to determine the relative levels of myocardial Cx43 mRNA transcripts in CF rabbits. Total RNA was extracted from rabbit left ventricle using REzol (PROtech, Taipei, ROC) according to manufacturer's protocol. In all, $1 \mu \mathrm{g}$ of total RNA was used for first-strand cDNA synthesis in 20- $\mu \mathrm{l}$ volumes using $1 \mathrm{mM}$ dNTPs (Amersham Biosciences, Buckinghamshire, UK), 100 U M-MLV 
reverse transcriptase, First-Strand Buffer $(50 \mathrm{mM}$ Tris-HCl, $75 \mathrm{mM} \mathrm{KCl,} 3 \mathrm{mM} \mathrm{MgCl}_{2}$ ), $10 \mathrm{mM}$ DTT (Invitrogen, Carlsbad, CA, USA), $0.2 \mu \mathrm{g}$ Oligo (dT) ${ }_{15}$ Primer, and $20 \mathrm{U}$ RNase inhibitor (Promega, Madison, WI, USA). For each RT product, aliquots $(2 \mu \mathrm{l})$ of the final reaction volume were amplified in two parallel PCR reactions with Cx43-specific (188-bp product, sense $5^{\prime}$-TCTGAGTGCCTGAACTTGC-3', antisense $\quad 5^{\prime}$-ACTGACAGCCACACCTTCC- $3^{\prime}$ ) or GAPDH-specific (545-bp product, sense 5'-CATT GACCTCAACTACATGG-3', antisense $5^{\prime}$-TTGCCCA CAGCCTTGGCAGC-3') primers. The PCR protocol comprised denaturation at $94^{\circ} \mathrm{C}$ for $10 \mathrm{~s}$, annealing at $62^{\circ} \mathrm{C}$ or $56^{\circ} \mathrm{C}$ for $10 \mathrm{~s}$, and elongation at $72^{\circ} \mathrm{C}$ for $30 \mathrm{~s}$ for the amplification of $\mathrm{Cx} 43$ and GAPDH cDNAs, respectively. Cx43 was amplified for 28 cycles and GAPDH for 21 cycles. RT-PCR products were analyzed by $2 \%$ agarose gel electrophoresis, visualized with ethidium bromide, scanned, and quantified by Imagemaster (Amersham Pharmacia Biotec, New Jersey, USA). Results are presented as ratios between $\mathrm{Cx} 43$ and GAPDH amplification analysis.

\section{Statistical Analysis}

Data are expressed as the mean value \pm s.d., and the data between groups, as well as each observation were tested for significance with ANOVA followed by a Bonferroni correction or an unpaired Student's $t$-test when appropriate. Statistical significance was assessed by a paired $t$-test when comparing echocardiographic parameters of the CW rabbits after dietary cholesterol withdrawal. A $P$-value $<0.05$ was considered significant.

\section{Results}

\section{Effects of Dietary Hypercholesterolemia on Cx43 Protein and Regulators of Cx43 Protein}

As shown in Table 1, feeding with a cholesterolenriched diet progressively increased the serum levels of triglyceride, and more prominently, cholesterol. The $\mathrm{CF}$ rabbits showed a statistically significant decrease of $21 \%(P=0.003)$ in $\mathrm{Cx} 43$ protein expression within 3 weeks (Figure 2b). The protein levels were further reduced at week 6 , and were about $40 \%$ of the controls after 9 weeks. The dietary cholesterol withdrawal for 18 weeks restored the Cx43 protein expression comparable to that at week 3 but less than that of the controls.

After feeding with cholesterol-enriched diet, the level of Cx43 transcripts was progressively elevated (ANOVA, $P<0.001$ ), showing a 3.5 -fold increase compared to the control at 12 weeks (Figure 3).

Table 1 Echocardiographic measurements after feeding with a cholesterol-enriched diet

\begin{tabular}{|c|c|c|c|c|c|}
\hline & \multicolumn{5}{|c|}{ Duration of feeding with a cholesterol-enriched diet } \\
\hline & Controls $(\mathrm{n}=20)$ & 3 weeks $(\mathrm{n}=25)$ & 6 weeks $(\mathrm{n}=20)$ & 9 weeks $(\mathrm{n}=15)$ & 12 weeks $(\mathrm{n}=10)$ \\
\hline Cholesterol (mg/dl) ${ }^{\dagger}$ & $65 \pm 8$ & $497 \pm 103^{\S}$ & $1554 \pm 164^{\S}$ & $2153 \pm 253^{\S}$ & $2835 \pm 258^{\S}$ \\
\hline Triglyceride $(\mathrm{mg} / \mathrm{dl})^{\dagger}$ & $64 \pm 8$ & $153 \pm 15^{\S}$ & $191 \pm 21^{\S}$ & $207 \pm 37^{\S}$ & $294 \pm 42^{\S}$ \\
\hline Heart rate (beats/min) & $149 \pm 14$ & $149 \pm 14$ & $147 \pm 28$ & $145 \pm 12$ & $157 \pm 19$ \\
\hline $\begin{array}{l}\text { Cyclic variations of integrated } \\
\text { backscatters }(\mathrm{dB})^{\dagger}\end{array}$ & $7.1 \pm 0.8$ & $6.9 \pm 0.8$ & $5.4 \pm 0.6^{\S}$ & $4.3 \pm 0.4^{\S}$ & $4.0 \pm 0.5^{\S}$ \\
\hline \multicolumn{6}{|l|}{$2 D$ echocardiography } \\
\hline LVEDD (mm) & $15.35 \pm 1.38$ & $15.30 \pm 1.27$ & $15.01 \pm 0.71$ & $14.73 \pm 1.43$ & $14.85 \pm 1.94$ \\
\hline FS (\%) & $26.3 \pm 4.1$ & $26.0 \pm 3.7$ & $27.7 \pm 2.9$ & $27.5 \pm 2.7$ & $25.0 \pm 4.4$ \\
\hline Anterior WT $(\mathrm{mm}) *$ & $1.99 \pm 0.10$ & $2.00 \pm 0.11$ & $2.04 \pm 0.13$ & $2.06 \pm 0.14$ & $2.13 \pm 0.11^{\ddagger}$ \\
\hline Posterior WT (mm)* & $2.00 \pm 0.11$ & $2.01 \pm 0.12$ & $2.05 \pm 0.14$ & $2.08 \pm 0.12$ & $2.13 \pm 0.12$ \\
\hline \multicolumn{6}{|l|}{ Doppler echocardiography } \\
\hline Peak aortic velocity $(\mathrm{cm} / \mathrm{s})$ & $70.7 \pm 12.5$ & $71.3 \pm 11.8$ & $68.9 \pm 8.4$ & $71.3 \pm 15.5$ & $66.3 \pm 8.7$ \\
\hline Aortic TVI (cm) & $7.18 \pm 1.35$ & $6.80 \pm 1.44$ & $7.09 \pm 1.03$ & $7.52 \pm 1.75$ & $6.46 \pm 0.80$ \\
\hline Peak E velocity $(\mathrm{cm} / \mathrm{s})$ & $50.3 \pm 8.8$ & $54.0 \pm 9.1$ & $52.7 \pm 8.2$ & $58.3 \pm 18.1$ & $47.1 \pm 11.2$ \\
\hline Peak A velocity (cm/s) & $31.1 \pm 6.9$ & $32.8 \pm 7.9$ & $32.7 \pm 12.5$ & $36.3 \pm 11.3$ & $31.2 \pm 10.3$ \\
\hline $\mathrm{E} / \mathrm{A}$ & $1.66 \pm 0.27$ & $1.73 \pm 0.42$ & $1.81 \pm 0.64$ & $1.78 \pm 0.86$ & $1.60 \pm 0.40$ \\
\hline Deceleration time (ms) & $68.9 \pm 13.7$ & $67.0 \pm 12.2$ & $68.1 \pm 16.6$ & $71.3 \pm 15.5$ & $70.8 \pm 16.6$ \\
\hline \multicolumn{6}{|l|}{ Tissue Doppler imaging } \\
\hline Lateral Sm $(\mathrm{cm} / \mathrm{s})^{\dagger}$ & $7.25 \pm 0.71$ & $6.88 \pm 0.88$ & $6.36 \pm 0.74^{\ddagger}$ & $6.14 \pm 1.16^{\ddagger}$ & $6.15 \pm 0.46^{\ddagger}$ \\
\hline Lateral Em $(\mathrm{cm} / \mathrm{s})$ & $8.16 \pm 0.96$ & $8.20 \pm 1.13$ & $7.88 \pm 0.50$ & $8.11 \pm 1.12$ & $7.37 \pm 1.11$ \\
\hline Lateral Am (cm/s) & $4.43 \pm 1.34$ & $4.20 \pm 0.89$ & $4.45 \pm 1.07$ & $4.39 \pm 1.43$ & $4.29 \pm 1.16$ \\
\hline Lateral Em/Am & $2.00 \pm 0.66$ & $2.02 \pm 0.45$ & $1.87 \pm 0.47$ & $1.99 \pm 0.55$ & $1.84 \pm 0.58$ \\
\hline Modified Tei index & $0.33 \pm 0.09$ & $0.37 \pm 0.09$ & $0.44 \pm 0.13^{\ddagger}$ & $0.54 \pm 0.14^{\S}$ & $0.56 \pm 0.10^{\S}$ \\
\hline E/Em & $6.23 \pm 1.28$ & $6.75 \pm 1.68$ & $6.70 \pm 1.04$ & $7.39 \pm 2.38$ & $6.47 \pm 1.65$ \\
\hline
\end{tabular}

E/A, ratio of peak early (E) and late (A) transmitral flow velocities; Em/Am, ratio of peak early (Em) and late (Am) diastolic velocities of mitral annulus; FS, fractional shortening; LVEDD, left ventricular end-diastolic diameter; $n$, rabbit number; Sm, peak systolic velocity of mitral annulus; TVI, time-velocity integral; WT, wall thickness in diastole.

${ }^{*} P<0.05$; ${ }^{\dagger} P<0.001$; by ANOVA. ${ }^{\ddagger} P<0.05 ;{ }^{\S} P<0.001$; vs control group with post hoc Bonferroni correction. 

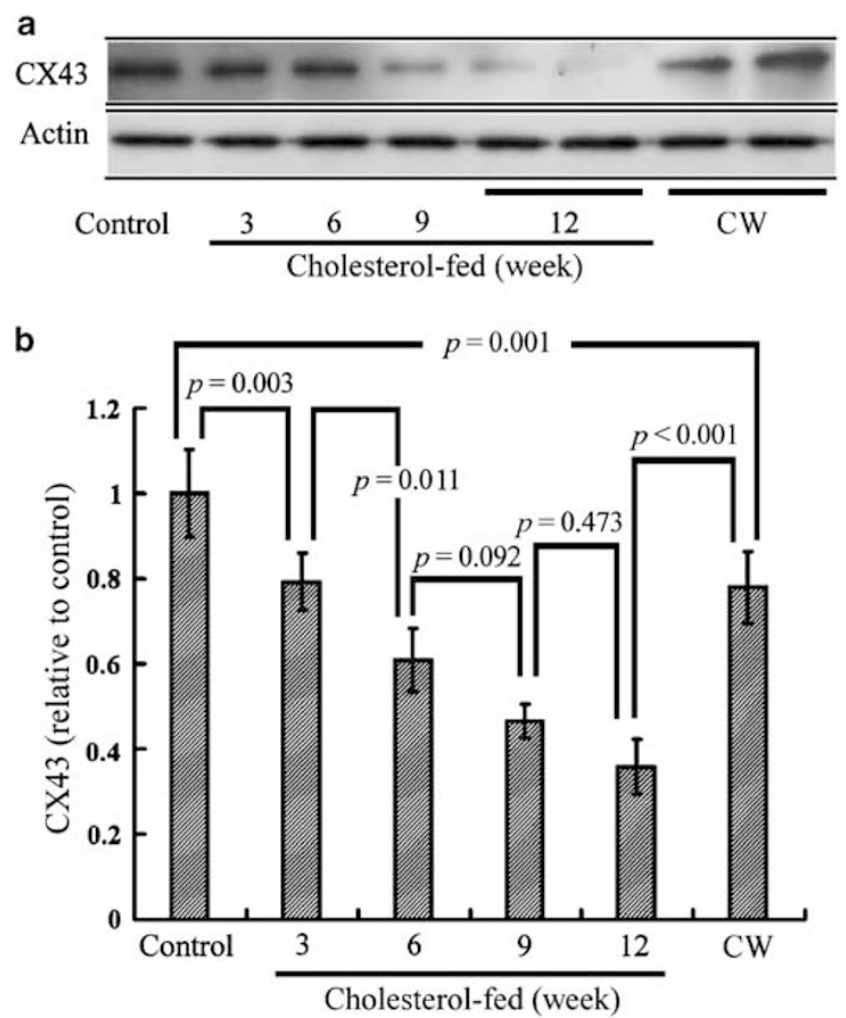

Figure 2 Immunoblots (a) and quantification in a bar diagram (b, $n=5$ animals each category) showing that $\mathrm{Cx} 43$ protein levels of cholesterol-fed rabbits significantly decreased within 3 weeks, progressively falling to $36 \%$ of the controls after feeding with a cholesterol-rich diet for 12 weeks. After dietary cholesterolwithdrawal (CW), the Cx43 protein levels were restored to comparable levels of that observed at 3 weeks. Differences between time points were tested with ANOVA with a post hoc Bonferroni correction. Data are mean \pm s.d. Blotting for $\alpha$-actin was performed on the same membrane to indicate relative loading.

Similarly, the level of phosphorylated JNK, which implies its activation, was also increased along the course of experiment (ANOVA, $P=0.001$; post hoc multiple comparison, $P=0.002$ between the group fed for 12 weeks and the control; Figure 4).

\section{Effects of Dietary Hypercholesterolemia on Cx43 Protein and Distribution of Gap Junctions}

The sequential immunoconfocal images of the CF rabbits demonstrated that the Cx43-labeling signals progressively and unevenly decreased and that there was a redistribution of gap junction plaques from the end-to-end intercalated disks to lateral cell borders (Figure 5b-e). The morphometric analyses showed that the $\mathrm{CF}$ rabbits had decreases in percentage area $(40 \%)$ occupied by Cx43 signals and gap junctional density (31\%), reaching statistical significance $(P<0.001)$ within 3 weeks $($ Figure $5 \mathrm{~g}$ and $\mathrm{h}$ ). Following the feeding protocol, the percentage area and density were further reduced

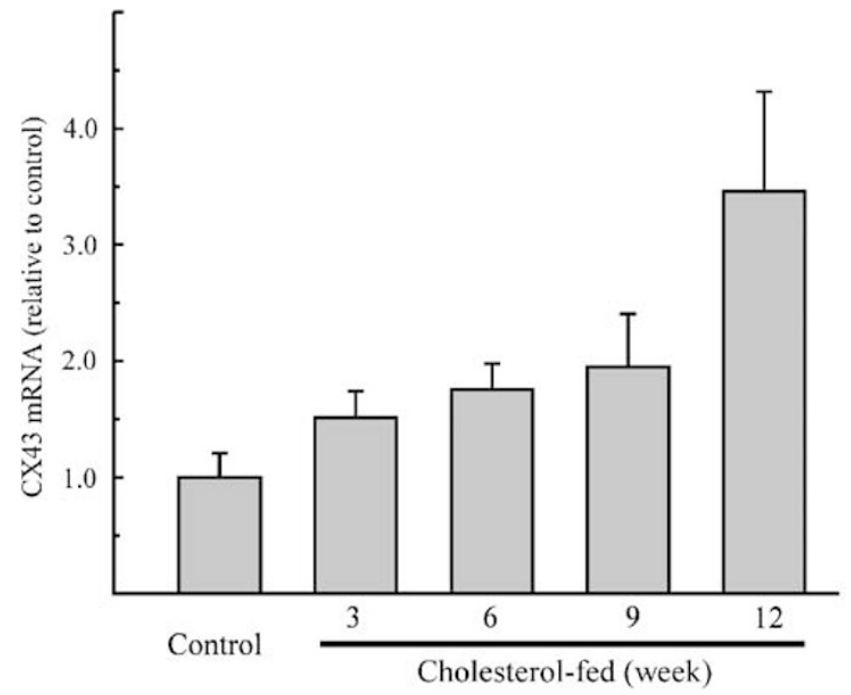

CX43

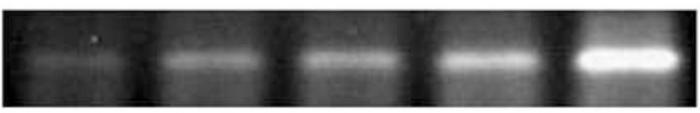

GAPDH

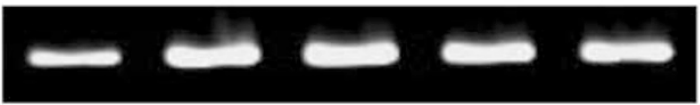

Figure 3 Upregulation of cardiac Cx43 transcripts after cholesterol-enriched diet, as demonstrated by semiquantitative RT-PCR. Note that the transcript level gradually increases during the course of the experiment (ANOVA, $P<0.001$ ). Each bar in the histogram above an example of gel represents the relative level (mean \pm s.d., $n=3$ ) of the amount of Cx43 transcripts in the corresponding lane, after normalization with GAPDH (below).

to $25 \%$ of controls for percentage area and $31 \%$ for density between nine and 12 weeks. The CW rabbits had the restored percentage area and density comparable to that of CF group at week 3 but less than the controls. Also, the distribution of gap junctions in CW rabbits had been restored to the end-to-end intercalated disk conformation (Figure 5f). The observed trend of alteration of elevated serum levels of cholesterol, in percentage area and density, was similar to that in $\mathrm{Cx} 43$ protein expression. However, the areas of immunolabeled Cx43 decreased significantly within three weeks after initiating a cholesterol-enriched diet, remaining stable throughout the following feeding and reinstating to comparable sizes of the controls after dietary cholesterol withdrawal (Figure 5i).

\section{Effects of Dietary Hypercholesterolemia on Myocardial Conduction Velocity}

The optical mapping data demonstrated that the intact ventricles of CF rabbits had slower conduction than the control (Figure 6c). The measured CVs $(\mathrm{cm} / \mathrm{s})$ for $\mathrm{CF}$ rabbits were significantly less than that of the control $(\mathrm{PCL}=200 \mathrm{~ms}, 50.62 \pm 0.86$ 

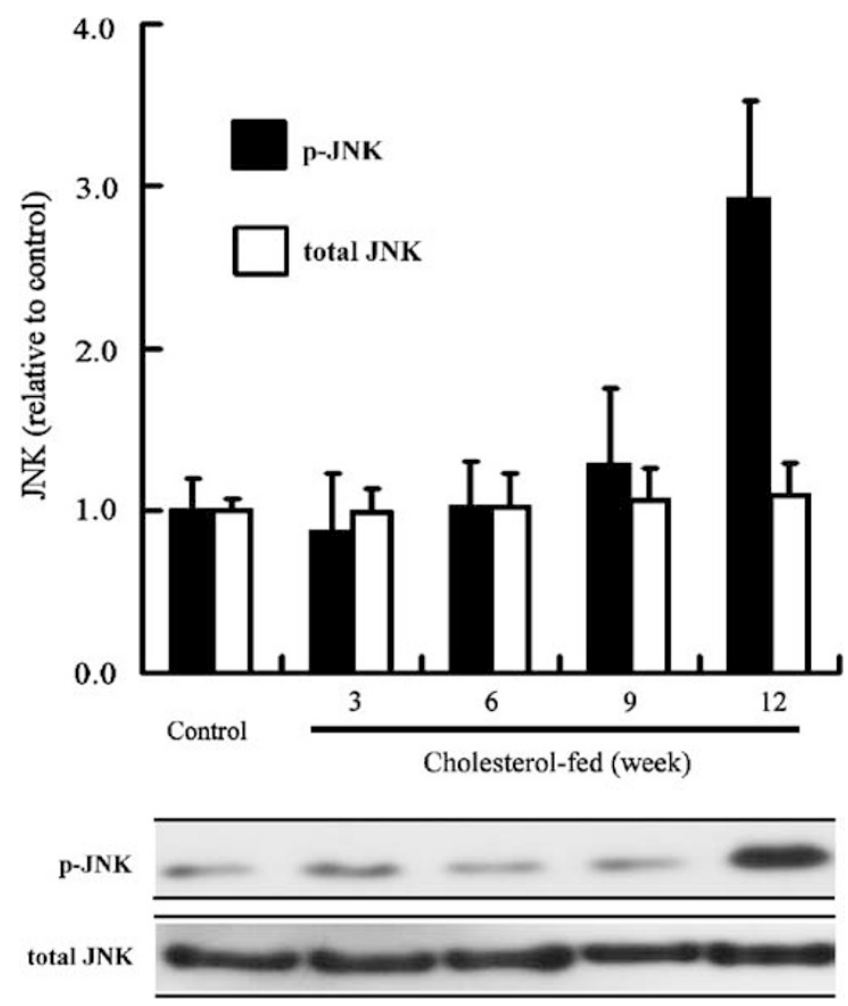

Figure 4 Immunoblots and quantification in a bar diagram showing static expression of total c-Jun $\mathrm{NH}_{2}$-terminal kinases (JNK) but increased level of its phosphorylated form (p-JNK; ANOVA, $P=0.001$ ) after feeding with a cholesterol-enriched diet Each bar in the histogram above the lanes of the gel represents the level normalized to the control (mean \pm s.d., $n=3$ ).

VS $57.50 \pm 1.55, P<0.001 ; 300 \mathrm{~ms}, 52.88 \pm 1.45$ Vs $59.11 \pm 1.64, \quad P<0.001 ; \quad 400 \mathrm{~ms}, \quad 52.88 \pm 1.45 \quad V S$ 59.30 $\pm 1.95, P=0.002$; Figure 6d).

\section{Histopathology and Hydroxyproline Content}

The hearts of CF rabbits had normal myocardial architectures without significant interstitial fibrosis (Figure $7 \mathrm{a}-\mathrm{d}$ ). The measured hydroxyproline content ( $\mathrm{g} / 100 \mathrm{~g}$ of frozen tissue), as the specific marker of collagen content in tissue, was similar in the groups of control $(0.026 \pm 0.006)$, CF $(0.023 \pm 0.006)$, and CW $(0.025 \pm 0.008)$ (ANOVA, $P=$ NS; Figure $7 \mathrm{e})$.

\section{Echocardiography after Cholesterol-Enriched Diet}

Compared with the controls, the CF rabbits had marginally thicker ventricular walls $(P=0.044)$, but with similar fractional shortening and ventricular diameter $(P=\mathrm{NS})$ after 12 weeks of enriched diet (Table 1). The peak velocity of the mitral annulus systolic wave $(\mathrm{Sm})$, proven to reflect the peak rate of the left ventricular pressure rise during contraction (peak dP/dt), ${ }^{29}$ decreased after 6 weeks of enriched diet $(P=0.016$, Figure $8 \mathrm{~b})$. The decrease in $\mathrm{Sm}$ was accompanied by an increase in modified Tei index
( $P=0.032$, Figure 8c), which implicated depressed ventricular mechanical performance. As the serum levels of cholesterol elevated, the modified Tei index further increased, stabilizing between weeks 9 and 12. Serial UTC of the CF rabbits (Figure 8a) demonstrated that the CVI remained unchanged through the initial 3 weeks but was significantly reduced $(P<0.001)$ by 6 weeks, progressively declining and also becoming stationary after 9 weeks of cholesterol-enriched diet. On the contrary, those parameters relating to ventricular diastolic function, such as E/A, deceleration time or Em/Am, were similar throughout the study.

\section{Echocardiography after Dietary Cholesterol Withdrawal}

The dietary cholesterol withdrawal for 18 weeks reverted the serum levels of total cholesterol $(56 \pm 11 \mathrm{mg} / \mathrm{dl})$ and triglyceride $(48 \pm 8 \mathrm{mg} / \mathrm{dl})$, similar to those of the controls $(P=\mathrm{NS})$, accompanied by the recovery of CVI (CW, after vs before dietary withdrawal, $7.7 \pm 0.6$ vs $4.4 \pm 0.2 \mathrm{~dB}, \quad P=0.002$, paired $t$-test) and modified Tei index $(0.44 \pm 0.04$ vs $0.53 \pm 0.04, P=0.034$, paired $t$-test) (Figure 8a and c). The fractional shortening remained unchanged $(24.6 \pm 2.4$ vs $24.4 \pm 3.5 \%, P=\mathrm{NS})$. Comparison of the other Doppler echocardiographic measurements revealed no significant difference (data not shown).

\section{Discussion}

In this study, we showed that diet-induced hypercholesterolemia in rabbits caused downregulation of myocardial Cx43 protein and redistribution of $\mathrm{Cx} 43$ gap junction, upregulation of $\mathrm{Cx} 43$ transcripts, and activation of JNK. The hypercholesterolemic rabbit hearts were also demonstrated to have systolic dysfunction in vivo, as measured using the CVI, mitral ring systolic velocity, and modified Tei index, all of which have been reported to be more sensitive than the fractional shortening. ${ }^{14,22,29}$ These findings of hyperlipidemia-induced protein and contractile change raised an interesting question regarding the relationship between the alterations.

Our findings suggest that in the hyperlipidemic rabbits, the remodeling of cardiomyocyte gap junctions may contribute to the impaired systolic function. Disturbances in the distribution of gap junctions and reduced levels of their major component, Cx43, have been associated with various cardiac diseases that exhibit contractile dysfunction. ${ }^{4-6}$ In the hibernating myocardium, the structural remodeling of gap junctions and reduced Cx43 protein expression has been found and implicated a possible link between disease-related gap junction remodeling and impaired cardiac contraction. ${ }^{8}$ The downregulation of $\mathrm{Cx} 43$ protein expression has also been demonstrated in the left ventricles of transplant patients with end-stage congestive heart 

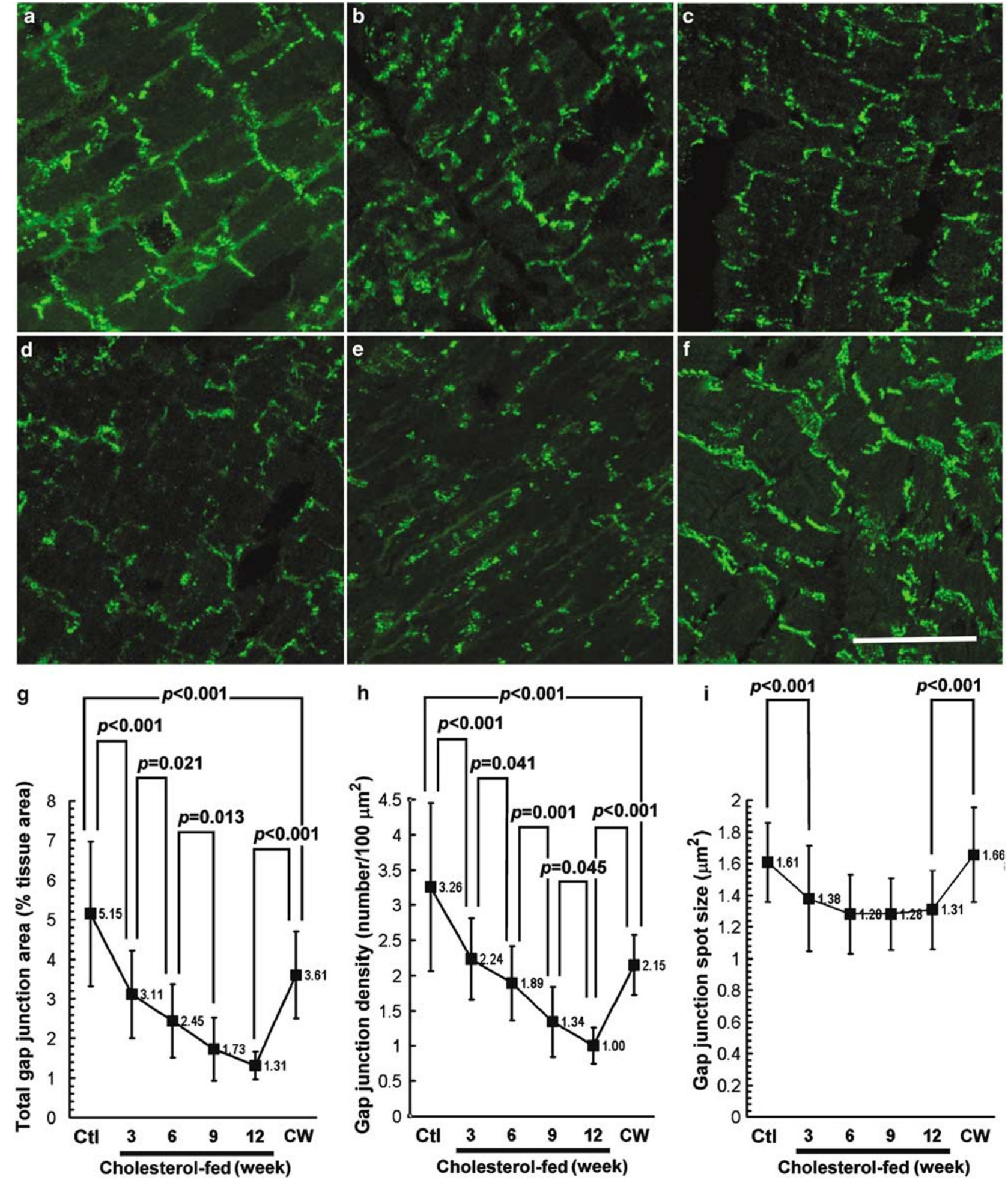

Figure 5 Immunoconfocal staining of Cx43 in myocardium of control (Ctl, a) as well as cholesterol-fed rabbits at weeks 3 (b), 6 (c), 9 (d), and 12 (e), demonstrating the remodeling of gap junctions in hypercholesterolemic hearts. Note that the progressive downregulation and redistribution of Cx43 signals reversed after dietary cholesterol-withdrawal (CW, f). The morphometric analyses of gap junctional plaques were also shown (g-i). See text for details. Bar represents $100 \mu \mathrm{m}$.

failure, irrespective of whether heart failure is due to idiopathic dilated cardiomyopathy or ischemic heart disease. ${ }^{9}$ Data from quantitative immunoconfocal microscopy of tissue from by-pass patients suggest that in ischemic heart disease, reduction in ventricular $\mathrm{Cx} 43$ develops long before terminal heart failure. ${ }^{30}$ The gap junction uncoupler, heptanol, has been reported to decrease left ventricular developed 
a

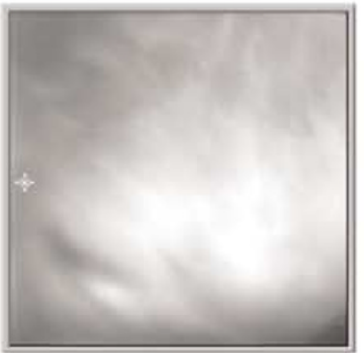

b

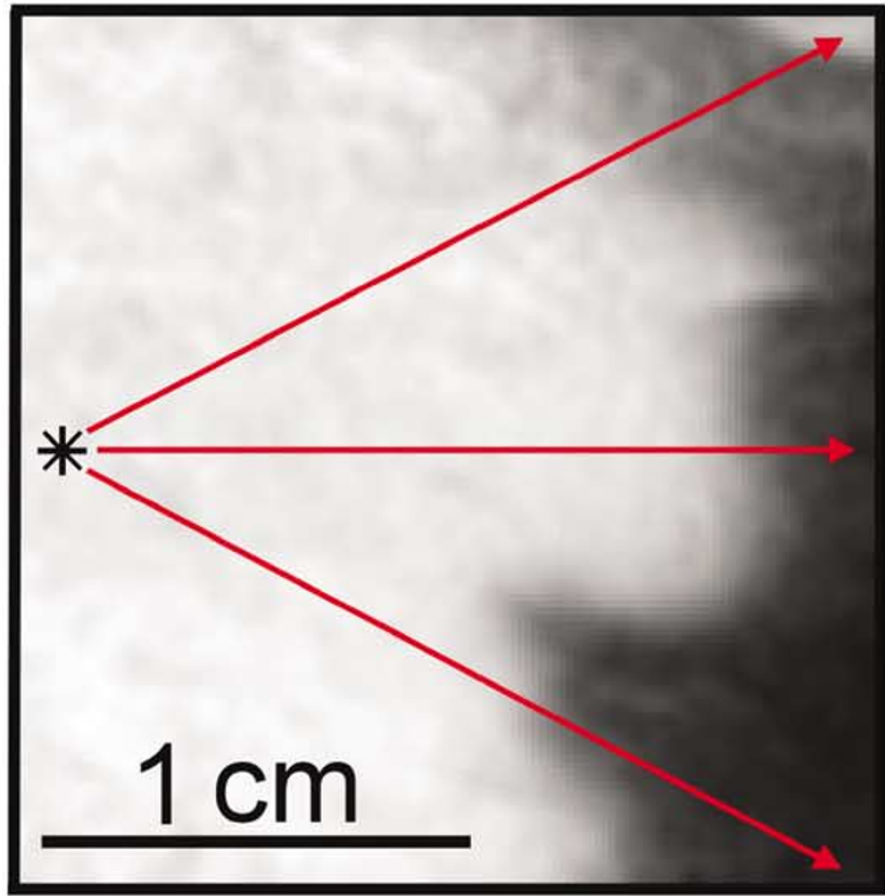

(31 ms)

C

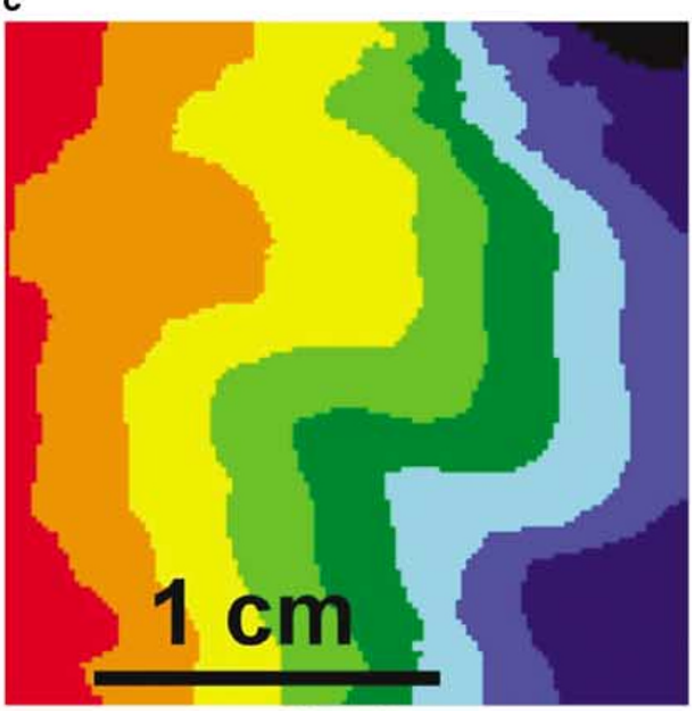

Control

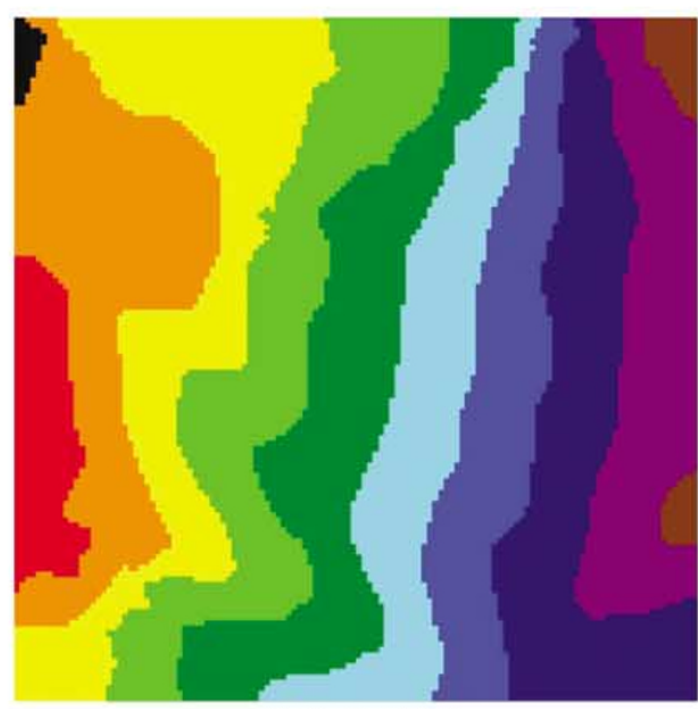

Cholesterol-fed

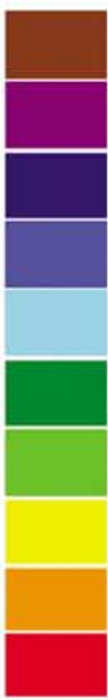

40.80

36.72

32.64

28.56

24.48

20.40

16.32

12.24

8.16

4.08

(msec)

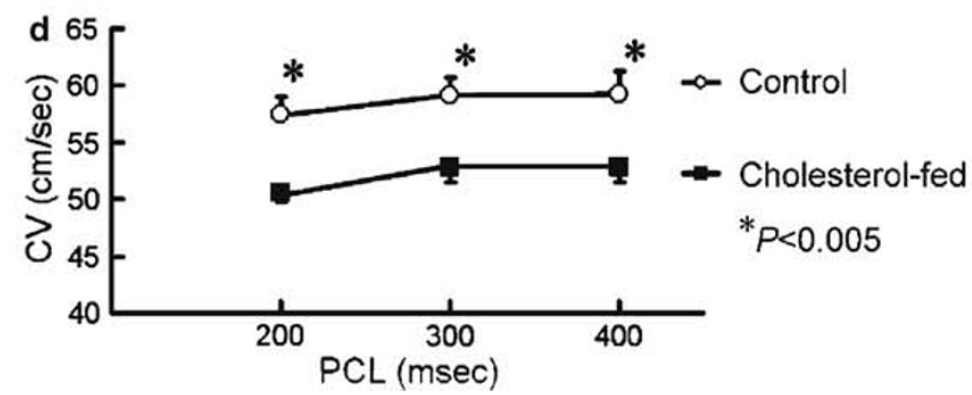


pressure in isolated perfused hearts. ${ }^{31}$ All of these data support our view that in the hyperlipidemic rabbits downregulation of cardiomyocyte gap junctions may contribute to the impaired systolic function.
Regarding the temporal association of the protein change and contractile dysfunction, the remodeling of gap junctions was significant within 3 weeks of cholesterol feeding when serum cholesterol levels were about $500 \mathrm{mg} / \mathrm{dl}$. However, no parameter
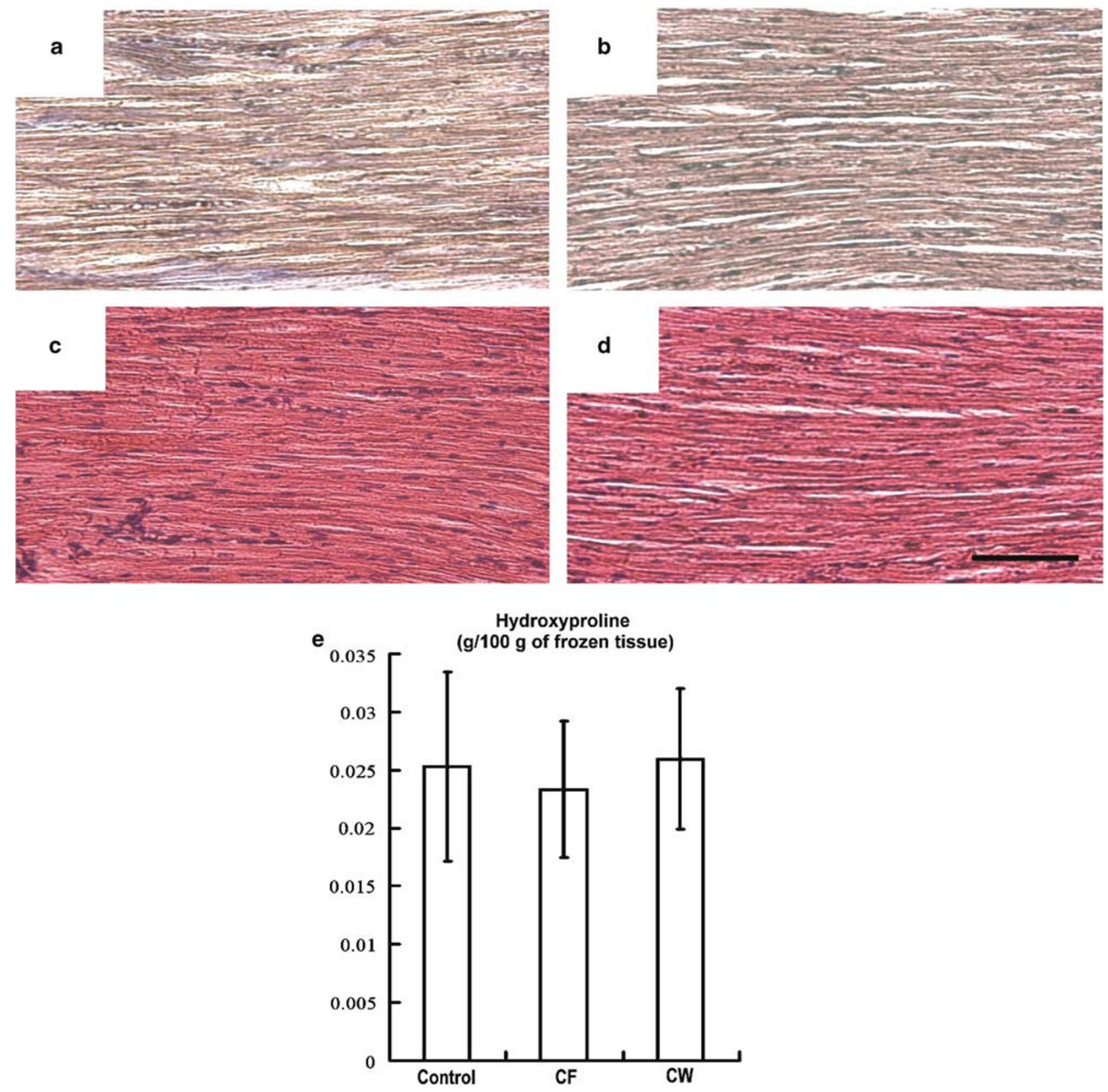

Figure 7 Histology of myocardium stained with Masson trichrome (a and $\mathbf{c}$ ), as well as hematoxylin and eosin (b and d). Note that the hypercholesterolemic hearts (c and $\mathbf{d}$ ) had a normal architecture and absence of interstitial fibrosis compared with the controls (a and b). (e) Quantification of hydroxyproline revealed similar results between groups of controls, cholesterol-fed (CF) rabbits for 12 weeks, and cholesterol-withdrawn (CW) rabbits for 18 weeks. Data are mean \pm s.d. Bar represents $100 \mu \mathrm{m}$.

Figure 6 Optical mapping for the determination of conduction velocity (CV). Voltage maps (a) show the progress of conduction wavefront at ventricular wall during pacing. Mean value of CV along the three long red arrows (b) was used. The isochronal maps (c) demonstrate the slowing of ventricular conduction in cholesterol-fed rabbit. (d) The measured CV of the cholesterol-fed rabbits $(n=3)$ was significantly less than that of the control $(n=6)$ among different pacing cycle lengths (PCL). Asterisks in (a) and (b) mark pacing site. 

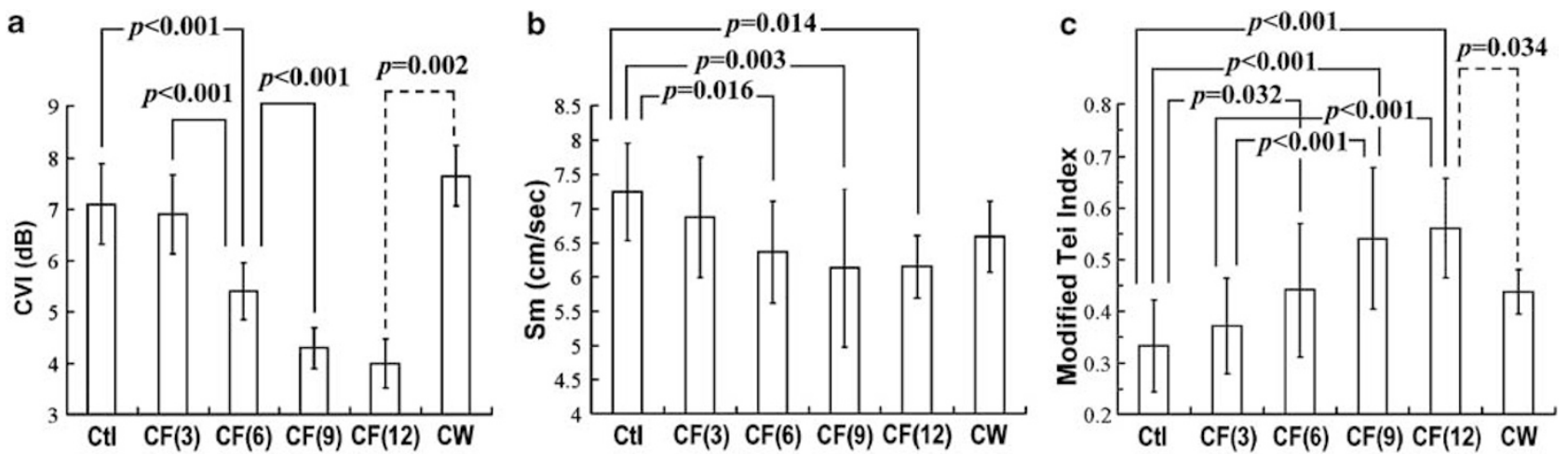

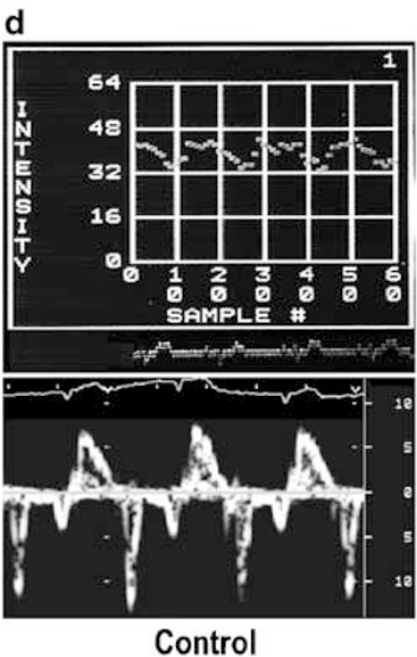

Control

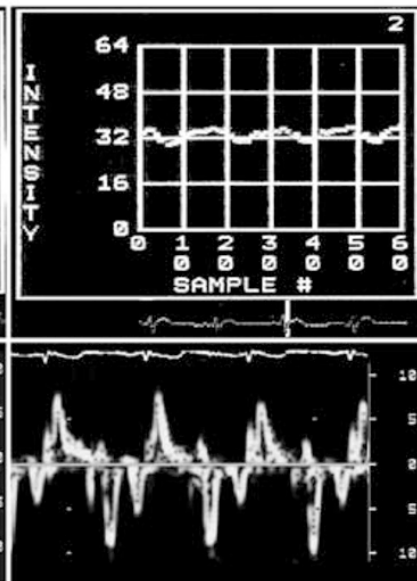

Cholesterol-fed

6 weeks

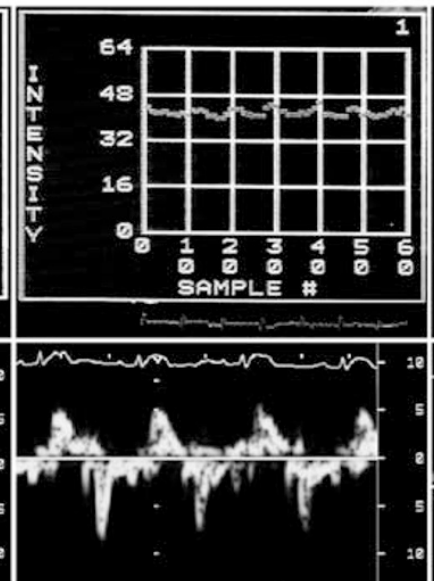

Cholesterol-fed 12 weeks

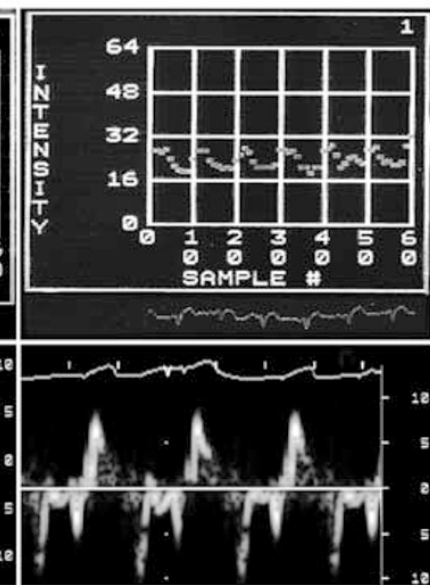

Cholesterol-withdrawal 18 weeks

Figure 8 (a) The cholesterol-fed (CF) rabbits showed progressively decreased cyclic variations of integrated backscatters (CVI) that reached significance $(P<0.001)$ at 6 weeks, stabilizing between 9 and 12 weeks (number in parenthesis indicating the duration of feeding with a cholesterol-enriched diet). The CVI was restored after dietary cholesterol-withdrawal (CW) for 18 weeks. (b) Peak velocities of the mitral annular systolic waves $(\mathrm{Sm})$ significantly decreased $(P<0.001)$ in CF rabbits after 6 weeks. (c) The modified Tei indexes of CF rabbits progressively increased $(P<0.001)$ after 6 weeks, becoming stationary between 9 and 12 weeks, and reversing after CW. (d) Typical echocardiography demonstrating the serial changes. Differences between these time points were tested with ANOVA with a post hoc Bonferroni correction (line) or a paired $t$-test (dashed line). Data are mean \pm s.d.

indicating contractile dysfunction was detected at this time period. After 6 weeks of cholesterol feeding, the serum cholesterol levels were elevated to about $1500 \mathrm{mg} / \mathrm{dl}$, and the $\mathrm{Cx} 43$ protein levels reduced to about $60 \%$ of the control values. The CVI attenuated, implicating the diminished intramural intrinsic contractility. The increases in the modified Tei index and the decreases in mitral ring systolic velocity also demonstrate the lessened ventricular force. The serum levels of cholesterol elevated as the feeding period extended. The alterations in CVI paralleled the structural remodeling of gap junctions and downregulation of $\mathrm{Cx} 43$ protein, which declined to a plateau after 9 weeks of cholesterol feeding. The end-systolic extent, revealed by fractional shortening in conventional B-mode echocardiogram, appeared unchanged throughout the feeding protocol. Meanwhile, the Doppler assessments in transmitral flow and tissue velocity did not reveal any sign of diastolic dysfunction. The dietary withdrawal of cholesterol reverted these alterations in Cx43 gap junctions and contractile dysfunction.
Gap junctions play an essential role in coupling adjacent cardiomyocytes, ensuring organized propagation of rhythmic action potential and synchronizing beating among cardiomyocytes. ${ }^{4,32}$ Considering the myocardium as an electromechanical syncytium, electrical heterogeneity and disrupted wave front propagation could in turn lead to uncoordinated myocyte contraction and diminish ventricular contractile force. Our study demonstrated the progressive remodeling of myocardial $\mathrm{Cx} 43$ and corresponding impairment of systolic function, along with the slowing of $\mathrm{CV}$, suggesting the linkage between gap junction and cardiac contractility.

The contractile dysfunction observed in the present study is consistent with the previous report that reduced maximum isotonic velocity and retarded maximum isometric force occurred in papillary muscles from hypercholesterolemic rabbits. ${ }^{11}$ The myocardial CVI, proven to be heart rate and load independent, could be considered as an index of intrinsic myocardial contractility. ${ }^{14,33}$ According to the elastic modules model ${ }^{13}$ and the microscopic 
elastic wave theory, ${ }^{16}$ the size and interdistance of scatters (myocytes), the acoustic impedance mismatching between the myocytes' intracellular contents and the extracellular collagen networks, as well as the ultrasonic insonification angle, ${ }^{34}$ determine the acoustic contrast responsible for the scattering and generate the CVI. In recent studies, CVI reduction has been considered as an early indicator of contractile dysfunction before recognizing the geometric remodeling or the drop in fractional shortening, being attributed to the increase or disarray in the myocardial collagen network. $^{14,35}$ However, the downregulation and redistribution of $\mathrm{Cx} 43$ has been also found in the hypertrophied myocardium, ${ }^{30}$ suggesting a possible role for the remodeling of gap junctions in the attenuated CVI. In this study, we reported the sequential alterations in contractile function and the expression and distribution of $\mathrm{Cx} 43$ in hearts of rabbits fed on cholesterol-enriched diet, that have normal myocardial architecture and ridding of transmural fibrosis, and also the reversal of these findings on withdrawal of the diet. The remodeled gap junctions abate local coordination and result in inhomogeneous myocytes' contraction that may reduce, by interfering, the magnitude of altering acoustic contrast during a cardiac cycle and accordingly, the CVI. The disorganization of intramural contraction could disperse the individual myocyte's effort and attenuate the entire contractile force as shown by the decreased Sm and impaired Tei index. Although the gap junction remodeling proceeded the statistically significant reduction in CVI, Sm and impairment of Tei index, the presentation of temporal profile may not be enough to establish a cause-effect relation between them, because the myosin isoform redistribution and downregulation of SERCA-2 have been observed in the failing myocardium. ${ }^{36}$ Further experiments will be required to disclose the critical role of contractile synchronism of normal myocytes in generating CVI.

Our optical mapping study demonstrated an approximately $10 \%$ decrease in $\mathrm{CV}$, associated with a more than $60 \%$ reduction in $\mathrm{Cx} 43$ protein level. It is comparable to the previous findings by Eloff et $a l^{37}$ that showed an approximately $50 \%$ reduction of Cx43 protein expression causing significant CV slowing in the mouse heart. ${ }^{37}$ The exposure to hypoxia also resulted in a $50 \%$ reduction of $\mathrm{Cx} 43$ protein expression and an approximately $20 \%$ decrease in CV of cultured neonatal rat cardiomyocytes. ${ }^{38}$ However, some recent investigations have shown that the slowing of impulse propagation depends on more severe reductions in Cx43 abundance. $^{3,39}$

The fluidity of cholesterol-rich domains in plasma membrane is important to gap junctional coupling. ${ }^{40}$ In our experimental animals, the membrane-enrichment by cholesterol will decrease the fluidity and impair the coupling. Besides, our previous study has shown that the dietary hypercholesterolemia pro- longed and dispersed the action potential durations of rabbit hearts. ${ }^{1}$ This electric remodeling may also contribute to the conduction slowing in the hypercholesterolemic myocardium. Using a genetically engineered murine model, Danik et $a l^{3}$ have demonstrated that uncoupling as a result of diminished Cx43 expression, in a heterogeneous fashion, plays a mechanical role in the formation of a highly arrhythmogenic substrate. We have not only shown the downregulated $\mathrm{Cx} 43$ protein expression but also the unevenly redistributed gap junctional plaques in these hypercholesterolemic hearts. Those alterations might have exaggerated nonhomogeneous resting potential and action potential upstroke velocity and duration, affecting the individual cell excitability and refractory period, dispersion of which is a key proarrhythmic factor. ${ }^{5,6}$

In this study, Cx43 transcripts were upregulated by dietary hypercholesterolemia, suggesting that the downregulation of $\mathrm{Cx} 43$ protein is due to post-transcriptional modification. The cholesterol enrichment of cell membranes after dietary hypercholesterolemia may alter the assembly and distribution of Cx43. ${ }^{41}$ Meyer et $a l^{42}$ have treated cells with cholesterol in serum-free media and observed a decrease in gap junction assembly. The dietary hypercholesterolemia, increasing nonspecific effectors of inflammation ${ }^{43}$ and the oxidative stressdependent processes of macrophages within the myocardium, ${ }^{44}$ was shown to activate JNK in this study. Activated JNK has been demonstrated to decrease Cx43, most likely at the post-transcriptional level, in cultured myocytes and in transgenic animal models. ${ }^{19}$ On the other hand, a wellcharacterized downstream targets of JNK, activating protein 1 is an activator of the $\mathrm{Cx} 43$ proximal promoter in several different cell types, including cardiomyocytes. ${ }^{45}$ Our study correlated, although with a time lag, the activation of JNK with the upregulation of Cx43 transcripts and downregulation $\mathrm{Cx} 43$ proteins. Further studies will be needed to establish the specific mechanisms of hypercholesterolemia-mediated Cx43 downregulation, including alterations in $\mathrm{Cx} 43$ protein synthesis, degradation and membrane assembly.

In conclusion, we have demonstrated that the association of the remodeling of gap junctions with the alterations of contractile function resulted from diet-induced hypercholesterolemia. Since gap junctions play a pivotal role in the coordinated excitation of the heart, their remodeling by hypercholesterolemia may partly explain the beneficial effects of lipid-lowering therapy in heart diseases.

\section{Acknowledgements}

This study was supported by the National Science Council (Taiwan) Grants NSC91-2314-B-002-283 and NSC92-2314-B-195-012, a National Taiwan 
University Hospital Grant NTUH92S024, and a Mackay Memorial Hospital Grant MMH-E-94003.

\section{References}

1 Liu YB, Wu CC, Lu LS, et al. Sympathetic nerve sprouting, electrical remodeling, and increased vulnerability to ventricular fibrillation in hypercholesterolemic rabbits. Circ Res 2003;92:1145-1152.

2 Gutstein DE, Morley GE, Tamaddon H, et al. Conduction slowing and sudden arrhythmic death in mice with cardiac-restricted inactivation of connexin 43. Circ Res 2001;88:333-339.

3 Danik SB, Liu F, Zhang J, et al. Modulation of cardiac gap junction expression and arrhythmic susceptibility. Circ Res 2004;95:1035-1041.

4 van Veen TAB, van Rijen HVM, Opthof T. Cardiac gap junction channels: modulation of expression and channel properties. Cardiovasc Res 2001;51: 217-229.

5 Severs NJ, Coppen SR, Dupont E, et al. Gap junction alterations in human cardiac disease. Cardiovasc Res 2004;62:368-377.

6 Severs NJ, Dupont E, Coppen SR, et al. Remodeling of gap junctions and connexin expression in heart disease. Biochim Biophys Acta 2004;1662:138-148.

7 Lerner DL, Beardslee MA, Saffitz JE. The role of altered intercellular coupling in arrhythmias induced by acute myocardial ischemia. Cardiovasc Res 2001;50: 263-269.

8 Kaprielian RR, Gunning M, Dupont E, et al. Downregulation of immunodetectable connexin43 and decreased gap junction size in the pathogenesis of chronic hibernation in the human left ventricle. Circulation 1998;97:651-660.

9 Dupont E, Matsushita T, Kaba RA, et al. Altered connexin expression in human congestive heart failure. J Mol Cell Cardiol 2001;33:359-371.

10 Gutstein DE, Morley GE, Vaidya D, et al. Heterogeneous expression of Gap junction channels in the heart leads to conduction defects and ventricular dysfunction. Circulation 2001;104:1194-1199.

11 Peterson DW, Griffith Jr DW, Napolitano CA. Decreased myocardial contractility in papillary muscles from atherosclerotic rabbits. Circ Res 1979;45:338-346.

12 Blumlein S, Sievers R, Wikman-Coffelt J, et al. Lack of decreased contractility in hearts from atherosclerotic rabbits. Am Heart J 1983;106:1383-1388.

13 Wickline SA, Thomas III LJ, Miller JG, et al. A relationship between ultrasonic integrated backscatter and myocardial contractile function. J Clin Invest 1985;76:2151-2160.

14 Di Bello V, Giorgi D, Talini E, et al. Incremental value of ultrasonic tissue characterization (backscatter) in the evaluation of left ventricular myocardial structure and mechanics in essential arterial hypertension. Circulation 2003;107:74-80.

15 Lin LC, Yen RF, Hwang JJ, et al. Ultrasonic tissue characterization evaluates myocardial viability and ischemia in patients with coronary artery disease. Ultrasound Med Biol 2000;26:759-769.

16 Rose JH, Kaufmann MR, Wickline SA, et al. A proposed microscopic elastic wave theory for ultrasonic backscatter from myocardial tissue. J Acoust Soc Am 1995;97:656-668.
17 Holland MR, Wilkenshoff UM, Finch-Johnston AE, et al. Effects of myocardial fiber orientation in echocardiography: quantitative measurements and computer simulation of the regional dependence of backscattered ultrasound in the parasternal short-axis view. J Am Soc Echocardiogr 1998;11:929-937.

18 Hoyt RH, Collins SM, Skorton DJ, et al. Assessment of fibrosis in infarcted human hearts by analysis of ultrasonic backscatter. Circulation 1985;71:740-744.

19 Petrich BG, Gong X, Lerner DL, et al. c-Jun N-terminal kinase activation mediates downregulation of connexin43 in cardiomyocytes. Circ Res 2002;91: 640-647.

$20 \mathrm{Wu}$ CC, Su MJ, Chi JF, et al. The effect of hypercholesterolemia on the sodium inward currents in cardiac myocyte. J Mol Cell Cardiol 1995;27:1263-1269.

21 Tei C, Nishimura RA, Seward JB, et al. Noninvasive Doppler-derived myocardial performance index: correlation with simultaneous measurements of cardiac catheterization measurements. J Am Soc Echocardiogr 1997;10:169-178.

22 Harada K, Tamura M, Toyono M, et al. Assessment of global left ventricular function by tissue Doppler imaging. Am J Cardiol 2001;88:927-932.

$23 \mathrm{Lin} \mathrm{LC,} \mathrm{Kao} \mathrm{HL,} \mathrm{Wu} \mathrm{CC,} \mathrm{et} \mathrm{al.} \mathrm{Alterations} \mathrm{of}$ myocardial ultrasonic tissue characterization by coronary angioplasty in patients with chronic stable coronary artery disease. Ultrasound Med Biol 2001;27: 1191-1198.

24 Yeh HI, Lai YJ, Lee SH, et al. Heterogeneity of myocardial sleeve morphology and gap junctions in canine superior vena cava. Circulation 2001;104: 3152-3157.

25 Stegemann H, Stalder K. Determination of hydroxyproline. Clin Chim Acta 1967;18:267-273.

26 Vozzi C, Dupont E, Coppen SR, et al. Chamber-related differences in connexin expression in the human heart. J Mol Cell Cardiol 1999;31:991-1003.

27 Severs NJ, Rothery S, Dupont E, et al. Immunocytochemical analysis of connexin expression in the healthy and diseased cardiovascular system. Microsc Res Tech 2001;52:301-322.

28 Pak HN, Oh YS, Liu YB, et al. Catheter ablation of ventricular fibrillation in rabbit ventricles treated with beta-blockers. Circulation 2003;108:3149-3156.

29 Sohn DW, Chung WY, Chai IH, et al. Mitral annulus velocity in the noninvasive estimation of left ventricular peak dP/dt. Am J Cardiol 2001;87:933-936.

30 Peters NS, Green CR, Poole-Wilson PA, et al. Reduced content of connexin43 gap junctions in ventricular myocardium from hypertrophied and ischemic human hearts. Circulation 1993;88:864-875.

31 Garcia-Dorado D, Inserte J, Ruiz-Meana M, et al. Gap junction uncoupler heptanol prevents cell-to-cell progression of hypercontracture and limits necrosis during myocardial reperfusion. Circulation 1997;96: 3579-3586.

32 Oyamada M, Kimura H, Oyamada Y, et al. The expression, phosphorylation, and localization of connexin 43 and gap-junctional intercellular communication during the establishment of a synchronized contraction of cultured neonatal rat cardiac myocytes. Exp Cell Res 1994;212:351-358.

33 Sagar KB, Pelc LE, Rhyne TL, et al. Influence of heart rate, preload, afterload, and inotropic state on myocardial ultrasonic backscatter. Circulation 1988;77: 478-483. 
34 Santarelli MF, Landini L. A model of ultrasound backscatter for the assessment of myocardial tissue structure and architecture. IEEE Trans Biomed Eng 1996;43:901-911.

35 Koyama J, Ray-Sequin PA, Falk RH. Prognostic significance of ultrasound myocardial tissue characterization in patients with cardiac amyloidosis. Circulation 2002;106:556-561.

36 Huang Y, Walker KE, Hanley F, et al. Cardiac systolic and diastolic dysfunction after a cholesterol-rich diet. Circulation 2004;109:97-102.

37 Eloff BC, Lerner DL, Yamada KA, et al. High resolution optical mapping reveals conduction slowing in connexin43 deficient mice. Cardiovasc Res 2001;51: 681-690.

38 Zeevi-Levin N, Barac YD, Reisner Y, et al. Gap junctional remodeling by hypoxia in cultured neonatal rat ventricular myocytes. Cardiovasc Res 2005;66:64-73.

39 van Rijen HV, Eckardt D, Degen J, et al. Slow conduction and enhanced anisotropy increase the propensity for ventricular tachyarrhythmias in adult mice with induced deletion of connexin43. Circulation 2004;109:1048-1055.
40 Bastiaanse EM, Jongsma HJ, van der Laarse A, et al. Heptanol-induced decrease in cardiac gap junctional conductance is mediated by a decrease in the fluidity of membranous cholesterol-rich domains. J Membr Biol 1993;136:135-145.

41 Malewicz B, Kumar VV, Johnson RG, et al. Lipids in gap junction assembly and function. Lipids 1990;25: 419-427.

42 Meyer RA, Lampe PD, Malewicz B, et al. Enhanced gap junction formation with LDL and apolipoprotein B. Exp Cell Res 1991;196:72-81.

43 Henry PD, Pacifico A. Altering molecular mechanisms to prevent sudden arrhythmic death. Lancet 1998;351: 1276-1278.

44 Kinscherf $\mathrm{R}$, Kamencic $\mathrm{H}$, Deigner $\mathrm{HP}$, et al. Hypercholesterolemia-induced long-term increase of macrophages in the myocardium of New Zealand White rabbits. Cells Tissues Organs 2003;174:184193.

45 Shyu KG, Wang BW, Yang YH, et al. Amphetamine activates connexin43 gene expression in cultured neonatal rat cardiomyocytes through JNK and AP-1 pathway. Cardiovasc Res 2004;63:98-108. 